\title{
Hypertension and beyond — does circulating irisin matter?
}

\author{
Aleksandra Taszarek, Anna Kaczmarkiewicz, Tomasz Miazgowski \\ Department of Hypertension and Internal Medicine, Pomeranian Medical University, Szczecin, Poland
}

\begin{abstract}
Irisin is a myokine secreted by skeletal muscles. It has been proposed to drive the brown-fat-like conversion of the white adipose tissue. By enhancing energy expenditure, irisin may affect systemic metabolism and be associated with insulin resistance; however, the mechanism(s) of action still remain largely unexplained. The discovery of irisin can contribute to the exploration of the novel and effective therapeutic targets or therapeutic strategies of metabolic diseases or metabolism-associated health issues. In this review, based on the recent literature (from 2013 to 2015 inclusive), we will systematically discuss the associations of irisin with obesity, type 2 diabetes, lipid disorders, and hypertension. We also introduce unanswered questions for future research.
\end{abstract}

Key words: irisin; hypertension; metabolic diseases; diabetes

Arterial Hypertens. 2016, vol. 20, no. 1, pages: 16-20

DOI: 10.5603/AH.2016.0004

\section{Background}

Irisin, which has been extensively studied since 2012 , is a cleaved and secreted fragment of the type I membrane of fibronectin type III domain-containing protein 5 (FNDC5) and is mainly secreted from skeletal muscle in response to exercise. The myokine induces browning of white adipose tissue and expends accumulated energy on the heat, and hence it was initially proposed to mediate the beneficial effects of exercising on energy metabolism and body composition. By this thermogenic effect, irisin improves metabolic profile of the adipose tissue and enhances whole-body energy expenditure. It was shown that on exercise stimulation, there is overexpression of FNDC5 (the precursor of irisin) in skeletal muscles, which acts as muscle-derived signal to the white adipose tissue $[1,2]$. As a result, irisin may promote increased oxygen consumption and energy supply [3]. Indeed, many earlier studies demonstrated association between intensive exercises and irisin levels.
Norheim et al. [4] studied the group of 26 inactive men (pre-diabetic and non-diabetic), aged 40-65 years, who were recruited for a 12-week intervention of combined endurance and strength training with four sessions of training per week. Before and after the 12-week intervention period, participants were exposed to an acute endurance workload of $45 \mathrm{~min}$ at $70 \%$ of $\mathrm{VO}_{2}$ max. They found a 1.2-fold increase in irsin levels shortly after acute endurance workloads; however, circulating irisin was reduced in response to 12 weeks of combined training. A similar, transient, post-load increase in irisin concentration was also reported in healthy individuals undergoing moderate aerobic exercises [5]. The brief rise in irisin level after exercise suggests an acute shedding event of irisin during exercise.

In contrast to those studies, several investigations failed to confirm these findings [6-9]. Kerstholt et al. found in the population-based Study of Health in Pomerania (SHIP-TREND) an inverse association

Address for correspondence: prof. Tomasz Miazgowski

Department of Hypertension and Internal Medicine, Pomeranian Medical University,

ul. Unii Lubelskiej 1, 71-252 Szczecin, tel. 91 4253550, fax: 914253552

e-mail: khchw@pum.edu.pl

V M Copyright () 2016 Via Medica, ISSN 2449-6170 
between irisin serum concentration and exercise capacity assessed by peak $\mathrm{VO}_{2}$ and maximum power output but only in men. In contrast, in women, a trend towards a positive relationship between irisin and peak $\mathrm{VO}_{2}$ was detected, whereas none of the other parameters showed significant associations with irisin [10]. Consistently, it was demonstrated that habitual runners had significantly healthier metabolic and body composition profiles compared with nonrunners; however, none of these favorable exercise effects were positively associated with circulating irisin [11].

Notwithstanding these discrepancies in the literature, irisin has gained great interest as a potential new marker, as well as a target to combat obesity and obesity-related metabolic abnormalities [12]. The rationale for this suggestion has been supported by experimental data. In the study of Saleem $e t$ al., $\mathrm{C} 57 \mathrm{Bl} / 6$ mice were fed with high-fat diet for 6 months until the animals were hyperglycemic and glucose intolerant. Then, intravenous injections of recombinant irisin were given for 8 weeks. Although treatment with irisin had no effect on fasting glucose levels or body weight, glucose tolerance in these animals significantly improved [13]. Other investigators described that central and peripheral irisin could differentially regulate cardiovascular activities. While administration of recombinant human irisin into the 3rd brain ventricle of rats activated neurons in the paraventricular nuclei of the hypothalamus with a subsequent increases in blood pressure (BP) and cardiac contractibility, peripheral administration of this myokine reduced BP in both control and spontaneously hypertensive rats [14]. The latter finding may be relevant in future investigations on potential therapeutic use of irisin in humans. However, studies evaluating the association of irisin with $\mathrm{BP}$ in humans are scarce.

\section{Irisin and blood pressure}

There have been only few reports evaluating the relation between circulating irisin and BP in humans. In a cross-sectional study, Yan et al. collected data on sociodemographic, lifestyle, and clinical characteristics from 1,115 community-living Chinese adults aged 52.3 years with central obesity [15]. Of them, $57 \%$ had raised BP defined as systolic BP $\geq 130 \mathrm{~mm} \mathrm{Hg}$ or diastolic $\mathrm{BP} \geq 85 \mathrm{~mm} \mathrm{Hg}$ or treatment of previously diagnosed hypertension. In this population, no significant associations between irisin level and BP were found. A similar lack of correlation of irisin with BP was found in a cohort of nondiabetic adult subjects [16].
In another cross-sectional study, Brondani et al. [17] investigated the association of FNDC5 rs3480 (A/G) and rs1746661 (G/T) polymorphisms with clinical characteristics in 1,006 patients with type 2 diabetes (T2DM) and 434 nondiabetic subjects. They found that the T allele of rs 1746661 was associated with increased systolic BP and not diastolic BP, but only in women with diabetes. In contrast to these reports, in 532 patients with stages $1-5$ of chronic kidney disease, irisin positively correlated with diastolic BP but not systolic BP [18]. However, Park et al. [19] demonstrated a positive correlation between irisin concentration and both systolic $\mathrm{BP}(\mathrm{R}=0.17 ; \mathrm{P}=0.04)$ and diastolic $\mathrm{BP}(\mathrm{R}=0.27 ; \mathrm{P}=0.001)$ in healthy middle-age individuals. The discrepancies in the abovementioned reports may be due to different populations analyzed in the studies, as some included subjects with metabolic diseases and wide ranges of BMI, which may influence baseline irisin levels. However, it is worth noting that to date none of the studies evaluated irisin levels in patients with essential hypertension and without coexisting diseases in comparison with normotensive subjects. Hence, in the light of promising results from rodent studies [14], clinical studies on irisin in patients with hypertension are urgently needed. Much more is known about altered irisin performance in conditions that frequently coexist with hypertension such as obesity, T2DM, and dyslipidemia.

\section{Irisin in obesity, diabetes and lipid disorders}

It has been shown in rodent models that overexpression of irisin increases total body energy expenditure, induces weight loss, and improves glucose tolerance and insulin sensitivity [20]. Moreover, in an experimental model, injected irisin alleviated endothelial dysfunction in diabetic rats by reducing oxidative/ nitrative stress through inhibiting signaling pathways implicating PKC- $\beta / \mathrm{NADPH}$ oxidase and NF- $\kappa \mathrm{B} /$ iNOS [21], as well as ameliorated lipid derangements via cAMP-PKA-HSL/perilipin pathway [22]. All together, these data suggest that irisin may be a promising molecule for the treatment of vascular complications and lipid disorders in T2DM.

In human studies, some evidence suggests an antidiabetic role of irisin. It was demonstrated that irisin may improve glucose homoeostasis $[23,24]$ and show an inverse association with intrahepatic triglyceride contents in obese [25]. However, contrary to these suggestions, obese individuals have been found to have higher circulating levels of irisin than 
Table I. Circulating irisin level in diabetes

\begin{tabular}{|l|l|l|}
\hline Type of diabetes & Irisin level & Reference \\
\hline Type 1 & Increased & Espes [35] \\
\hline Long-term T2DM & Decreased & Moreno-Navarette [31]; Liu [37]; Zhang [38]; Duran [39]; Assyov [40] \\
\hline Newly diagnosed T2DM & Decreased & Choi [32]; Xiang [36] \\
\hline Gestational diabetes & $\begin{array}{l}\text { Decreased } \\
\text { No difference }\end{array}$ & $\begin{array}{l}\text { Yuksel [41]; Kuzmicki [42]; Wawrusiewicz [43]; Zhao [44] } \\
\text { Ebert [45] }\end{array}$ \\
\hline
\end{tabular}

normal weight or anorexic patients [26, 27]. Moreover, higher irisin levels were also reported in individuals with metabolic syndrome (MetS) compared to those without MetS [19] as well as nondiabetic adults with insulin resistance and evidences of vascular atherosclerosis [16]. Reinforcing these findings, significant weight loss, either by calorie restricted diet or bariatric surgery, has been demonstrated to lower circulating irisin level, while the reduction of irisin after weight loss was reversed in those subjects who regained their original weight $[28,29]$. On the other hand, some studies reported no correlation or decreased irisin levels in obese compared to non-obese individuals $[30,31]$ or even a negative correlation of irisin with body mass index (BMI) [32].

Despite these controversies, most studies demonstrated a positive association of circulating irisin level with obesity suggesting that plasma irisin concentrations reflect net body adiposity, which is in apparent conflict with the suggested anti-obesity effect of this myokine. A possible explanation which could reconcile the discrepant data may be that FNDC5/irisin in physiological conditions exert a protective effect against obesity mediated by the browning of white adipose tissue and is thus increased in compensation for increasing body mass. In states of severe obesity, irisin in physiological concentration is not capable to maintain the balance of energy storage and energy expenditure. In such situation, along with muscle, irisin is also generated by adipose tissue [27], responding to direct alterations in body fat mass. Therefore, the increased circulating levels of irisin observed in obese patients may represent an adaptive response to counteract the metabolic disturbances associated with obesity [33, 34] and likely, when this adaptive mechanism fails, target tissues develop resistance to irisin. If confirmed, stimulation of irisin secretion or release through pharmacological strategies or use of irisin as a therapeutic agent could both prove ineffective, at least in extremely obese individuals.

Plasma irisin level in diabetes varies depending on the type of disease. There has been only one study demonstrating that in type 1 diabetes irisin level is increased [35], while in long-term [31, 37-40] and newly diagnosed T2DM $[32,36]$, and gestational diabetes it is frequently decreased in comparison with normal glucose tolerance (Table I). Assyov et al. in a well-designed study, demonstrated that circulating irisin levels progressively decreased with the worsening of glucose tolerance: it was the highest in individuals with normal glucose tolerance (median value of $619 \mathrm{ng} / \mathrm{ml}$; interquartile range (IQR) $=567 \mathrm{ng} / \mathrm{ml})$, lower in prediabetes $(314 \mathrm{ng} / \mathrm{ml}$; IQR $=577 \mathrm{ng} / \mathrm{ml})$ and the lowest in T2DM $(228 \mathrm{ng} / \mathrm{ml}$; $\mathrm{IQR}=200 \mathrm{ng} / \mathrm{ml}$ ) [40], suggesting that with progression of insulin resistance, plasma irisin levels diminish. On the other hand, although in their study, irisin correlated negatively with fasting glucose $(\mathrm{R}=-0.547$; $\mathrm{p}<0.001)$, the authors showed a positive correlation between irisin and BMI $(\mathrm{R}=0.475$; $\mathrm{p}<0.001)$.

Lowered values of irisin in T2DM may seem surprising as subjects with this condition are frequently overweight or obese and therefore they rather should tend to have higher irisin levels. Additionally, they develop insulin resistance, which is also frequently associated with increased irisin levels [19]. A possible explanation of this paradox might be that the main source of circulating irisin in extremely obese subjects without overt glucose dysregulation seems to be the adipose tissue and not skeletal muscles [27]. In turn, in nonobese to moderately obese patients with insulin resistance, irisin released from muscles might be downregulated due to muscle insulin resistance, although to date, studies have yielded ambiguous results. Recent reports have described a direct association between irisin and insulin, alongside with an inverse correlation between irisin and ghrelin (orexigenic hormone with an opposite action to insulin) [29], while intervention studies showed that after treatment designed to lose weight, higher irisin concentrations at the beginning of the intervention were associated with greater reductions in glucose, insulin and irisin concentrations, and homoeostasis model assessment of insulin resistance (HOMA-IR) index, independent of body weight reduction [46]. Irisin was identified as a potential factor associated with progression of insulin resistance in patients who re- 
Table II. Correlation between irisin and serum lipids

\begin{tabular}{|l|l|l|}
\hline Lipids & Correlation with irisin & Reference \\
\hline Total cholesterol & Positive & Liu [37]; Tang [48] \\
& Negative & Huh [28]; Iglesia [49] \\
\hline Low density lipoproteins & Positive & Iglesia [48]; Liu [36] \\
& Negative & Tang [48] \\
\hline High density lipoproteins & Positive & Weng [50] \\
& Negative & Park [19] \\
\hline Triglycerides & None & Iglesia [48] \\
& Positive & Liu [37]; Park [19] \\
\hline
\end{tabular}

gain their diet-induced weight loss [47]. Accordingly, a positive correlation has been found between baseline irisin levels and insulin resistance, as assessed by HOMA-IR [19]. However, whether decreased irisin level in T2DM represents a true cause-effect relationship remains unclear.

Irisin has also been tested as a marker of abnormal lipid profile, which gave unfavorable cardiovascular outcomes but results have been inconsistent [48-52]. Studies have shown either positive or negative associations of irisin with total cholesterol, high (HDL) and low density lipoproteins (LDL), and triglycerides (Table II). Zhang et al. [25] found that serum irisin levels were reduced in obese adults with non-alcoholic fatty liver disease (NAFLD), progressively with the increase of intrahepatic triglyceride content. These findings strongly suggest that irisin may play an important role in lipid metabolism and the development of NALFD. In obese individuals with other cardiovascular risk factors, increased circulating irisin levels were found to be correlated with unfavorable lower HDL [51]. On the other hand, lower irisin level was an independent predictor of macrovascular complications in T2DM [38] and similarly, in non-diabetic population - a robust predictor of unwilling cardiovascular outcomes such as myocardial infarction [52].

\section{Concluding remarks}

Irisin has been primarily investigated as a key myokine, which has a bridging potential between muscle and fat. Growing evidence supports the hypothesis that increased circulating irisin may reflect a compensatory response to impaired insulin sensitivity and other metabolic disorders associated with obesity. However, the potentially beneficial effect of FNDC5/irisin in the treatment of hypertension and obesity-related metabolic deteriorations has been challenged by insufficient and contradictory findings. Albeit preliminary results of treatment with exogenous irisin of hypertension and glucose intolerance in rodents are promising, it is too early to translate the effects observed in murine models to humans as they need to be confirmed in further studies aiming to (i) identify irisin receptor; (ii) understand the molecul ar pathways regulating irisin expression; and (iii) establish causality of this myokine in the development of metabolic diseases. In addition, as human FNDC5 is mainly translated from its non-canonical start codon, some recent reports even questioned the existence of circulating irisin and claimed that many human irisin antibodies used in commercial ELISA kit lack required specificity $[1,34]$.

\section{References}

1. Jedrychowski M.P., Wrann C.D., Paulo J.A. et al. Detection and quantitation of circulating human irisin by tandem mass spectrometry. Cell Metab. 2015; 22: 734-740.

2. McMillan A.C., White M.D. Induction of thermogenesis in brown and beige adipose tissues: molecular markers, mild cold exposure and novel therapies. Curr. Opin. Endocrinol. Diabetes Obes. 2015; 22: $347-352$.

3. Erickson H.P. Irisin and FNDC5 in retrospect: an exercise hormone or a transmembrane receptor? Adipocyte 2013; 2: 289-293.

4. Norheim F., Langleite T.M., Hjorth M. et al. The effects of acute and chronic exercise on PGC-1alpha, irisin and browning of subcutaneous adipose tissue in humans. FEBS J. 2014; 281: 739-749.

5. Kraemer R.R., Shockett P., Webb N.D. et al. A transient elevated irisin blood concentration in response to prolonged, moderate aerobic exercise in young men and women. Horm. Metab. Res. 2014; 46: 150-154.

6. Kurdiova T., Balaz M., Vician M. et al. Effects of obesity, diabetes and exercise on Fndc5 gene expression and irisin release in human skeletal muscle and adipose tissue: in vivo and in vitro studies. J. Physiol. 2014; 592: 1091-1107.

7. Moraes C., Leal V.O., Marinho S.M. et al. Resistance exercise training does not affect plasma irisin levels of hemodialysis patients. Horm. Metab. Res. 2013; 45: 900-904.

8. Pekkala S., Wiklund P.K., Hulmi J.J. et al. Are skeletal muscle FNDC5 gene expression and irisin release regulated by exercise and related to health? J. Physiol. 2013; 591: 5393-5400.

9. Hecksteden A., Wegmann M., Steffen A. et al. Irisin and exercise training in humans - results from a randomized controlled training trial. BMC Med. 2013; 11: 235.

10. Kerstholt N., Ewert R., Nauck M. et al. Association of circulating irisin and cardiopulmonary exercise capacity in healthy volunteers: results of the Study of Health in Pomerania. BMC Pulm. Med. 2015; 15: 41.

11. Hew-Butler T., Landis-Piwowar K., Byrd G. et al. Plasma irisin in runners and nonrunners: no favorable metabolic associations in humans. Physiol. Rep. 2015; 3: e12262. 
12. Chen J.Q., Huang Y.Y., Gusdon A.M., Qu S. Irisin: a new molecular marker and target in metabolic disorder. Lipids Health Dis. 2015; 14: 2.

13. Saleem A., Safdar A., Haikalis M. et al. Exercise-induced amelioration of diet-induced obesity and diabetes is not regulated by irisin. FASEB J. 2015; 29: 992.4.

14. Zhang W., Chang L., Zhang C. et al. Central and peripheral irisin differentially regulate blood pressure. Cardiovasc. Drugs Ther. 2015; 29: 121-127.

15. Yan B., Shi X., Zhang H. et al. Association of serum irisin with metabolic syndrome in obese Chinese adults. PLoS One. 2014; 9: e94235.

16. Sesti G., Andreozzi F., Fiorentino T. et al. High circulating irisin levels are associated with insulin resistance and vascular atherosclerosis in a cohort of nondiabetic adult subjects. Acta Diabetol. 2014; 51: 705-713.

17. Brondani L.A., Boelter G., Assmann T.S. et al. Irisin-encoding gene (FNDC5) variant is associated with changes in blood pressure and lipid profile in type 2 diabetic women but not in men. Metabolism 2015; 64: 952-957.

18. Ebert T., Focke D., Petroff D. et al. Serum levels of the myokine irisin in relation to metabolic and renal function. Eur. J. Endocrinol. 2014; 170: 501-506.

19. Park K.H., Zaichenko L., Brinkoetter M. et al. Circulating irisin in relation to insulin resistance and the metabolic syndrome. J. Clin. Endocrinol. Metab. 2013; 98: 4899-4907.

20. Liu T.Y., Shi C.X., Gao R. et al. Irisin inhibits hepatic gluconeogenesis and increases glycogen synthesis via the PI3K/Akt pathway in type 2 diabetic mice and hepatocytes. Clin. Sci. (Lond). 2015; 129: 839-850.

21. Zhu D., Wang H., Zhang J. et al. Irisin improves endothelial function in type 2 diabetes through reducing oxidative/nitrative stresses. J. Mol. Cell Cardiol. 2015; 87: 138-147.

22. Xiong X.Q., Chen D., Sun H.J. et al. FNDC5 overexpression and irisin ameliorate glucose/lipid metabolic derangements and enhance lipolysis in obesity. Biochim. Biophys. Acta 2015; 1852: 1867-1875.

23. Hojlund K., Bostrom P. Irisin in obesity and type 2 diabetes. J. Diab. Compl. 2013; 27: 303-304.

24. Vamvini M.T., Aronis K.N., Panagiotou G. et al. Irisin mRNA and circulating levels in relation to other myokines in healthy and morbidly obese humans. Eur. J. Endocrinol. 2013; 169: 829-834.

25. Zhang H.J., Zhang X.F., Ma Z.M. et al. Irisin is inversely associated with intrahepatic triglyceride contents in obese adults. J. Hepatol. 2013; 59: 557-562.

26. Stengel A., Hofmann T., Goebel-Stengel M. et al. Circulating levels of irisin in patients with anorexia nervosa and different stages of obesity — correlation with body mass index. Peptides. 2013; 39: 125-130.

27. Pardo M., Crujeiras A.B., Amil M. et al. Association of irisin with fat mass, resting energy expenditure, and daily activity in conditions of extreme body mass index. Int. J. Endocrinol. 2014; 857270.

28. Huh J.Y., Panagiotou G., Mougios V. et al. FNDC5 and irisin in humans: I. Predictors of circulating concentrations in serum and plasma and II. mRNA expression and circulating concentrations in response to weight loss and exercise. Metabolism 2012; 61: 1725-1738.

29. Crujeiras A.B., Pardo M., Arturo R.R. et al. Longitudinal variation of circulating irisin after an energy restriction-induced weight loss and following weight regain in obese men and women. Am. J. Hum. Biol. 2014; 26: 198-207.

30. Sanchis-Gomar F., Alis R., Pareja-Galeano H. et al. Circulating irisin levels are not correlated with BMI, age, and other biological parameters in obese and diabetic patients. Endocrine 2014; 46: 674-677.

31. Moreno-Navarrete J.M., Ortega F., Serrano M. et al. Irisin is expressed and produced by human muscle and adipose tissue in association with obesity and insulin resistance. J. Clin. Endocrinol. Metab. 2013; 98: E769-778.
32. Choi Y.K., Kim M.K., Bae K.H. et al. Serum irisin levels in new-onset type 2 diabetes. Diabetes Res Clin Pract. 2013; 100: 96-101.

33. Chen J.Q., Huang Y.Y., Gusdon A.M., Qu S. Irisin: a new molecular marker and target in metabolic disorder. Lipids Health Dis. 2015; 14: 2 .

34. Crujeiras A.B., Pardo M., Casanueva F.F. Irisin: 'fat' or artefact. Clin. Endocrinol. 2015; 82: 467-474.

35. Espes D., Lau J., Carlsson P.O. Increased levels of irisin in people with long-standing type 1 diabetes. Diabet. Med. 2015; 32: 1172-1176.

36. Xiang L., Xiang G., Yue L. et al. Circulating irisin levels are positively associated with endothelium-dependent vasodilation in newly diagnosed type 2 diabetic patients without clinical angiopathy. Atherosclerosis 2014; 235: 328-333.

37. Liu J.J., Wong M.D., Toy W.C. et al. Lower circulating irisin is associated with type 2 diabetes mellitus. J. Diabetes Compl. 2013; 27: 365-369.

38. Zhang M., Chen P., Chen S. et al. The association of new inflammatory markers with type 2 diabetes mellitus and macrovascular complications: a preliminary study. Eur. Rev. Med. Pharmacol. Sci. 2014; 18: 1567-1572.

39. Duran I.D., Gülçelik N.E., Ünal M. et al. Irisin levels in the progression of diabetes in sedentary women. Clin. Biochem. 2015; 48: 1268-1272.

40. Assyov Y., Gateva A., Tsakova A., Kamenov Z. Irisin in glucose continuum. Exp. Clin. Endocrinol. Diabetes 2016; 124: 22-27.

41. Yuksel M.A., Oncul M., Tuten A. et al. Maternal serum and fetal cord blood irisin levels in gestational diabetes mellitus. Diabetes Res. Clin. Pract. 2014; 104: 171-175.

42. Kuzmicki M., Telejko B., Lipinska D. et al. Serum irisin concentration in women with gestational diabetes. Gynecol. Endocrinol. 2014; 30: 636-639.

43. Wawrusiewicz-Kurylonek N., Telejko B., Kuzmicki M. et al. Increased maternal and cord blood betatrophin in gestational diabetes. PLoS ONE. 2015; 10: e0131171.

44. Zhao L., Li J., Li Z.L. et al. Circulating irisin is lower in gestational diabetes mellitus. Endocr. J. 2015; 62: 921-926.

45. Ebert T., Stepan H., Schrey S. et al. Serum levels of irisin in gestational diabetes mellitus during pregnancy and after delivery. Cytokine 2014; 65: 153-158.

46. Lopez-Legarrea P., de la Iglesia R., Crujeiras A.B. et al. Higher baseline irisin concentrations are associated with greater reductions in glycemia and insulinemia after weight loss in obese subjects. Nutr. Diabetes. 2014; 4: e110.

47. Crujeiras A.B., Zulet M.A., Lopez-Legarrea P. et al. Association between circulating irisin levels and the promotion of insulin resistance during the weight maintenance period after a dietary weight-lowering program in obese patients. Metabolism 2014; 63: 520-531.

48. Tang S., Zhang R., Jiang F. et al. Circulating irisin levels are associated with lipid and uric acid metabolism in a Chinese population. Clin. Exp. Pharmacol. Physiol. 2015 Jun 26. doi: 10.1111/14401681.12439. [Epub ahead of print].

49. Iglesia R., Lopez-Legarrea P., Crujeiras A.B. et al. Plasma irisin depletion under energy restriction is associated with improvements in lipid profile in metabolic syndrome patients. Clin. Endocrinol. 2014; 81: 306-311.

50. Wen M.S., Wang C.Y., Lin S.L., Hung K.C. Decrease in irisin in patients with chronic kidney disease. PLoS One. 2013; 8: e64025.

51. Panagiotou G., Mu L., Na B. et al. Circulating irisin, omentin-1, and lipoprotein subparticles in adults at higher cardiovascular risk. Metabolism. 2014; 63: 1265-1271.

52. Emanuele E., Minoretti P., Pareja-Galeano H. et al. Serum irisin levels, precocious myocardial infarction, and healthy exceptional longevity. Am. J. Med. 2014; 127: 888-890. 